\title{
Web-based Supplementary Materials
}

\section{Variance Function Estimation in Quantitative Mass Spectrometry with Application to iTRAQ Labeling}

\author{
Micha Mandel* Manor Askenazi ${ }^{\dagger}$ Yi Zhang and Jarrod A. Marto $^{\S}$
}

June 3, 2012

*Department of Statistics, The Hebrew University of Jerusalem, Mount Scopus, Jerusalem, Israel, 91905 (msmic@huji.ac.il) phone: 972-2-5883303, fax:972-2-5883549

${ }^{\dagger}$ Departments of Cancer Biology and Blais Proteomics Center, Dana-Farber Cancer Institute, and Department of Biological Chemistry and Molecular Pharmacology, Harvard Medical School, Boston, MA 02215-5450, and Department of Biological Chemistry, The Hebrew University of Jerusalem, Israel.

${ }_{\ddagger}^{\ddagger}$ Departments of Cancer Biology and Blais Proteomics Center, Dana-Farber Cancer Institute, Boston, MA $02215-5450$.

$\S$ Departments of Cancer Biology and Blais Proteomics Center, Dana-Farber Cancer Institute, and Department of Biological Chemistry and Molecular Pharmacology, Harvard Medical School, Boston, MA 02215-5450 


\section{A Workflow of the iTRAQ Technique}

The iTRAQ technique is a labelling scheme that supports up to 8 (but more commonly 4) distinct labels allowing the concurrent measurement of multiple biological samples in a single experimental run. The typical workflow is shown in Figure 1. Four different biological samples are first enzymatically digested to yield peptides which are then labelled using the various iTRAQ reagents. The peptide samples are then mixed and consequently subjected to identical handling throughout a standard mass-spectrometry based proteomics workflow. This involves separation of peptides using liquid chromatography (LC) followed by mass spectrometry (MS) analysis. An initial MS scan is generated, to identify the masses of peptides eluting from the LC column, which are then selected and subjected to tandem mass spectrometry (MS/MS), yielding a series of MS/MS spectra per peptide. The process is repeated yielding thousands of MS/MS spectra. Each individual spectrum corresponds to a peptide with the majority of peaks used to identify the amino-acid sequence of the peptide. However, a small portion of the spectrum yields iTRAQ reporter ions whose relative abundance is used to infer the relative abundance of the given peptide in the original samples. An estimate of the ratio of abundance for a given peptide between e.g. Sample 2 and Sample 1, will correspond to the ratio of the i115 and i114 peak intensities (so called because their masses are 114 and 115 daltons, respectively). 

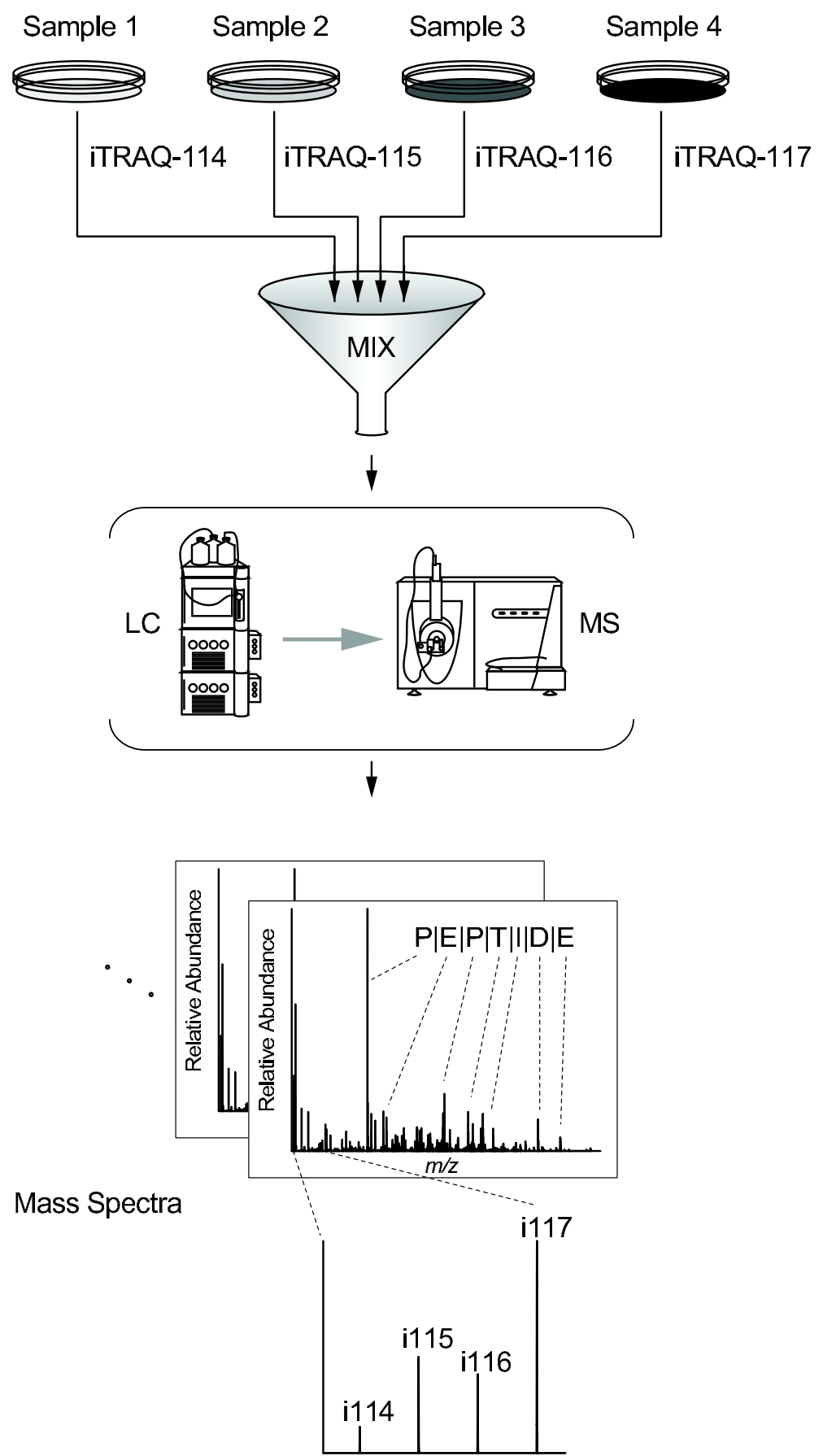

Figure 1: Four peptide samples are labeled, mixed and subjected to liquid chromatography (LC) followed by mass spectrometry (MS) yielding thousands of peptide mass spectra providing measurements of relative abundance by mass-to-charge ratio $(\mathrm{m} / \mathrm{z})$. The peak pattern can be used as a fingerprint identifying an individual peptide, and the relative peak height of the label reporter ions (i114-i117) provide relative abundance measurements of the peptide in the different samples. 


\section{B Estimate of $G_{0}$}

The EM algorithm estimated both the variance parameter $\theta$ and the distribution of the peptides amount $G_{0}$. Figure 2 displays the estimated $G_{0}$ using the pooled data of the control experiments. We considered a discrete distribution on $J=230$ support points determined as described in Section 2.3 of the main paper. The left panel presents the estimated cumulative distribution function and the right panel displays an histogram with width 0.25 of each category. The distribution spread over the support quite uniformly with two modes around 9 and 11.5.
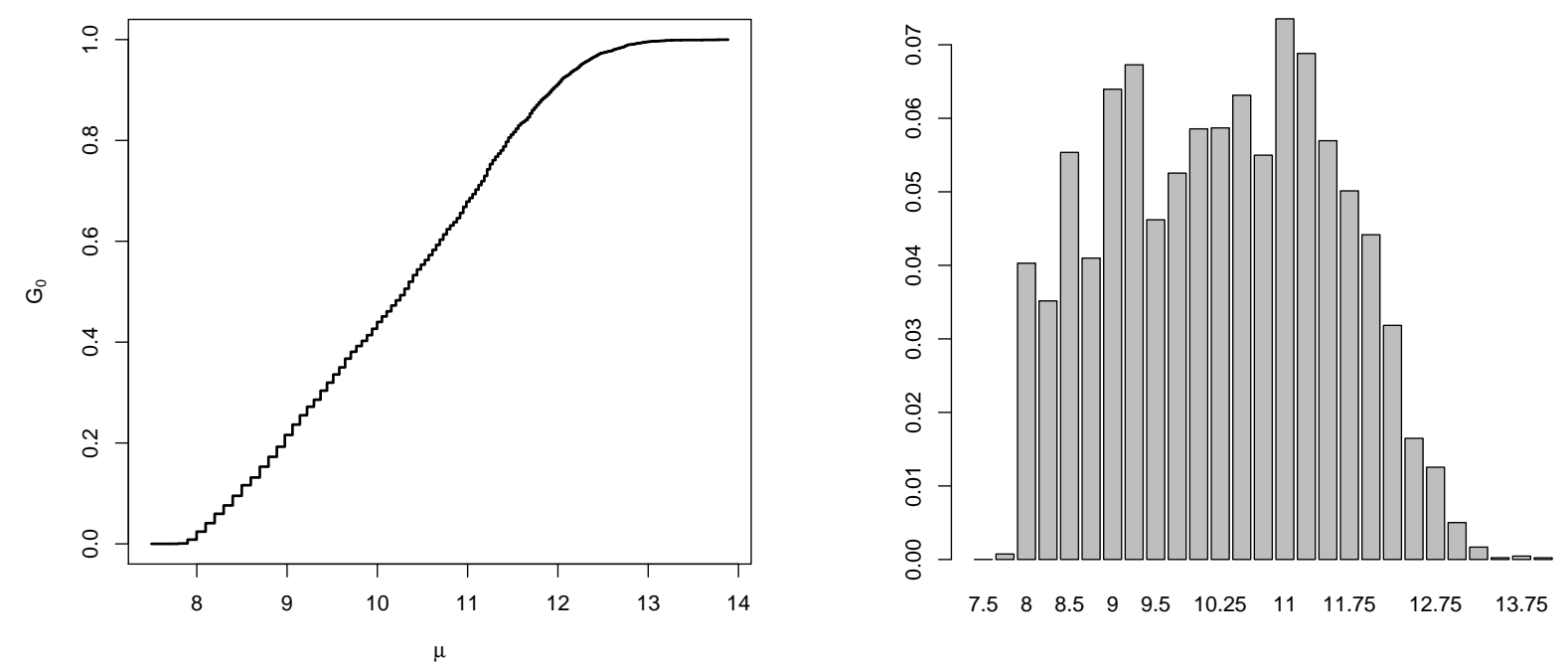

Figure 2: Estimated distribution of the peptide amounts in the control experiments. 


\section{Sensitivity of the EM Algorithm to Initial Values}

Initial values for the EM algorithm were based on the MACL approach applied to the March data $\left(\theta_{1}=4.89, \theta_{2}=-0.935\right)$. In order to test the effect of the initial values on the results, we recalculated the estimates starting from 20 random points that were sampled from the rectangular region $[4.89-1,4.89+1] \times[-0.935-0.5,-0.935+0.5]$. The 20 initial points (black circles) and the resulting estimates for the March (blue dots) and January (red dots) data are shown in the left panel of Figure 3. The right panel of the figure presents the variance functions $\exp \left(\theta_{1}+\theta_{2} \mu\right)$ corresponding to the initial values (black dotted lines) and to the March (blue lines) and January (red lines) data. The variance function estimates from all starting points are practically identical and cannot be distinguished.
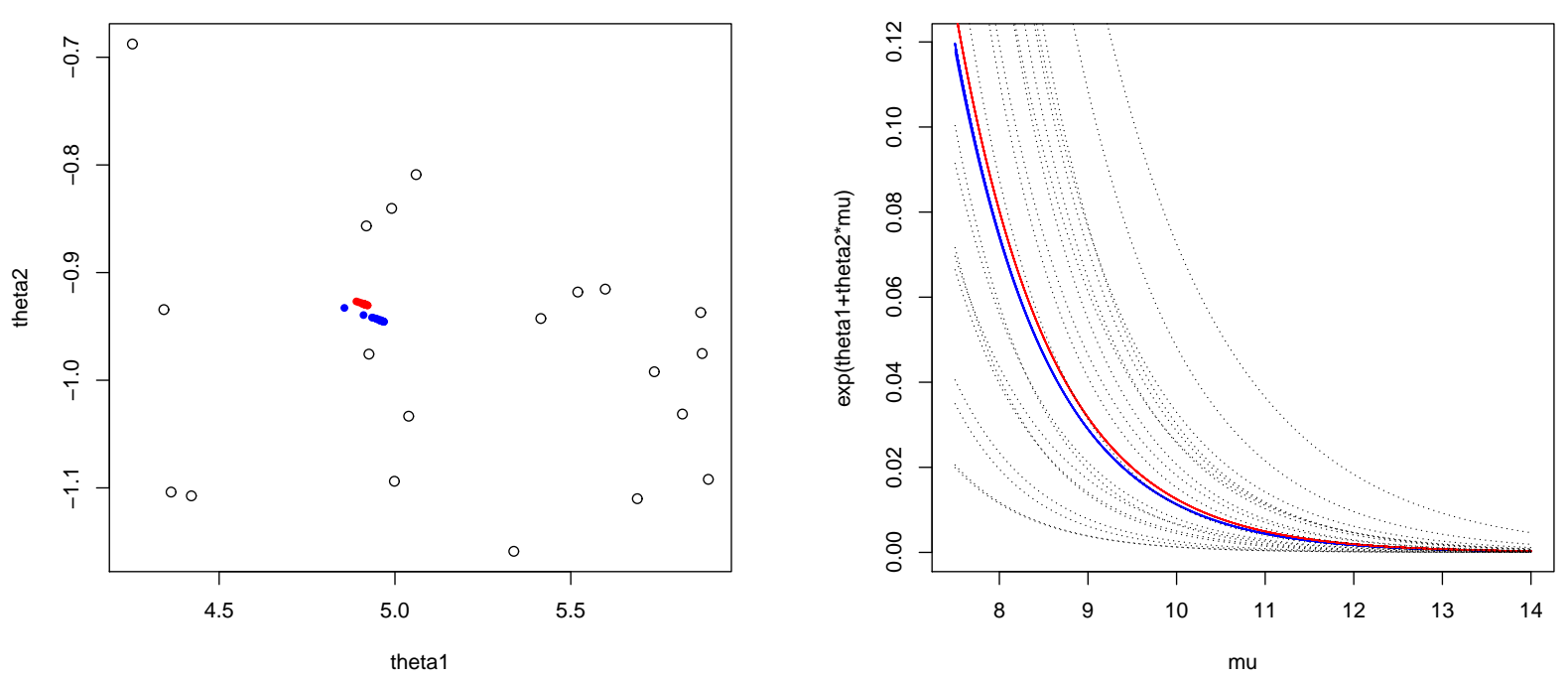

Figure 3: Sensitivity of the EM to initial values. 


\section{Simulation results}

\begin{tabular}{lrrrcrrr}
\hline & & \multicolumn{2}{c}{$\theta_{1}$} & & $\theta_{2}$ \\
\multicolumn{1}{c}{$\mu$} & $N$ & $\theta_{1}$ & bias & std & $\theta_{2}$ & bias & std \\
\hline Obs fixed & 200 & 5 & -0.061 & 0.830 & -1 & 0.006 & 0.081 \\
Obs fixed & 500 & 5 & -0.051 & 0.508 & -1 & 0.005 & 0.049 \\
Obs fixed & 1000 & 5 & -0.020 & 0.360 & -1 & 0.002 & 0.036 \\
Obs fixed & 2000 & 5 & -0.002 & 0.251 & -1 & 0.000 & 0.025 \\
\hline Obs random & 200 & 5 & 0.009 & 0.785 & -1 & -0.001 & 0.078 \\
Obs random & 500 & 5 & -0.023 & 0.519 & -1 & 0.003 & 0.051 \\
Obs random & 1000 & 5 & -0.011 & 0.338 & -1 & 0.001 & 0.033 \\
Obs random & 2000 & 5 & -0.015 & 0.251 & -1 & 0.002 & 0.025 \\
\hline $\mathrm{U}(8,12)$ & 200 & 5 & 0.026 & 0.858 & -1 & -0.003 & 0.085 \\
$\mathrm{U}(8,12)$ & 500 & 5 & -0.016 & 0.566 & -1 & 0.002 & 0.056 \\
$\mathrm{U}(8,12)$ & 1000 & 5 & -0.014 & 0.400 & -1 & 0.002 & 0.040 \\
$\mathrm{U}(8,12)$ & 2000 & 5 & -0.029 & 0.271 & -1 & 0.003 & 0.027 \\
\hline $\mathrm{U}\{8,9, \ldots, 12\}$ & 200 & 5 & 0.041 & 0.707 & -1 & -0.004 & 0.067 \\
$\mathrm{U}\{8,9, \ldots, 12\}$ & 500 & 5 & -0.001 & 0.460 & -1 & 0.000 & 0.043 \\
$\mathrm{U}\{8,9, \ldots, 12\}$ & 1000 & 5 & 0.001 & 0.324 & -1 & 0.000 & 0.031 \\
$\mathrm{U}\{8,9, \ldots, 12\}$ & 2000 & 5 & -0.012 & 0.220 & -1 & 0.001 & 0.021
\end{tabular}

Table 1: Simulation results for the MACL method - comparison of different distributions $G_{0}$ for the variance function $e^{5-\mu}$. 

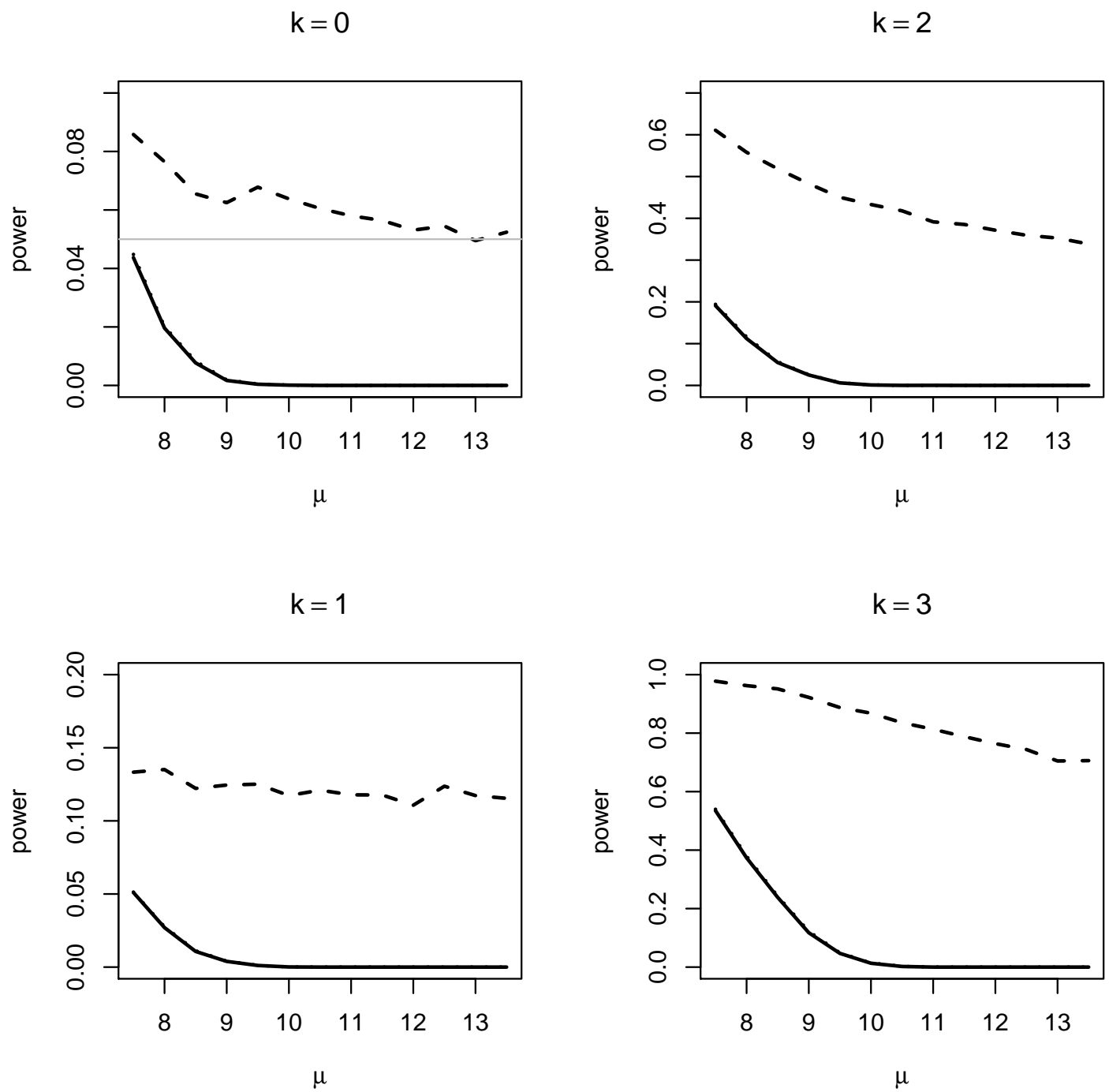

Figure 4: Power function for testing $\mu_{1}=\mu_{2}$ when $Y_{1} \sim N\left(\mu, e^{5-0.5 \mu}\right)$ and $Y_{2} \sim N\left(\mu_{k}, e^{5-0.5 \mu_{k}}\right)$, where $\mu_{k}=\mu+k \times \sqrt{e^{\theta_{1}+\theta_{2} \mu}}$ ( $k=0$ corresponds to the null hypothesis). The graphs compare the power of the naive (dashed), Berger and Boos (solid) and conservative (dotted) approaches, for different alternatives, $\mu_{2}=\mu_{1}+k \times \sqrt{e^{\theta_{1}+\theta_{2} \mu_{1}}} \quad k=0,1,2,3$. Tests are of level 0.05 . 

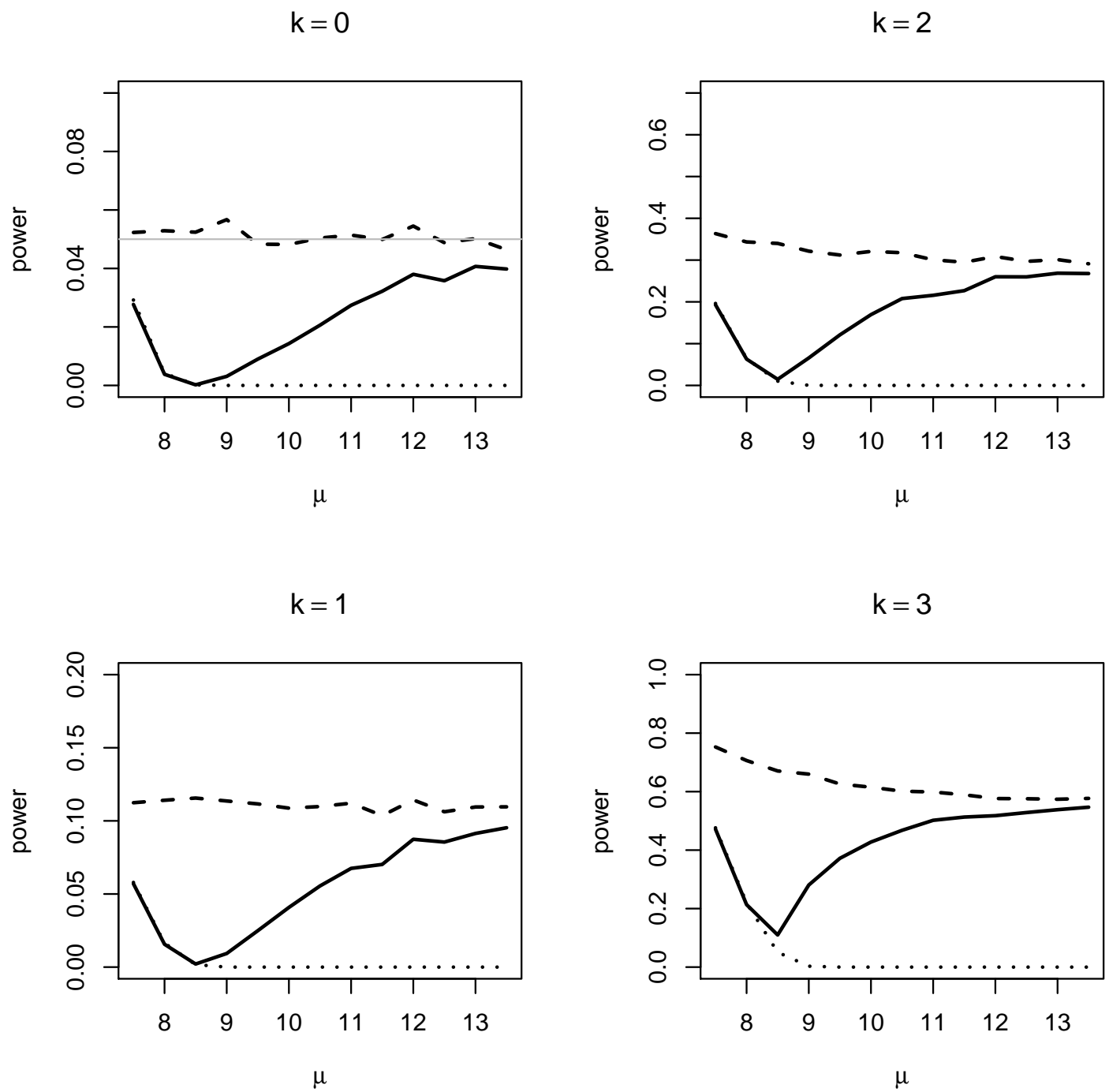

Figure 5: Power function for testing $\mu_{1}=\mu_{2}$ when $Y_{1} \sim N\left(\mu, e^{5-\mu}\right)$ and $Y_{2} \sim N\left(\mu_{k}, e^{5-\mu_{k}}\right)$, where $\mu_{k}=\mu+k \times \sqrt{e^{\theta_{1}+\theta_{2} \mu}}$ ( $k=0$ corresponds to the null hypothesis). The graphs compare the power of the naive (dashed), Berger and Boos (solid) and conservative (dotted) approaches, for different alternatives, $\mu_{2}=\mu_{1}+k \times \sqrt{e^{\theta_{1}+\theta_{2} \mu_{1}}} \quad k=0,1,2,3$. Tests are of level 0.05 . 